\title{
EL MITO DEL POLÍTICO (268 D-274 E) Y LA HISTORIA DE LAS RELIGIONES
}

The importance of a religious-historical approach for an appropriate understanding of the myth in Plato's Statesman has not been sufficiently emphasized yet. The myth substantially describes the life of the world as two revolutions of the same circle, the one forward and the other backward. In the forward revolution God accompanies the world; during the latter he remains in his watch-tower. Within the framework of this myth, which establishes the current philosophical doctrine of the alternating world-periods, we find more or less developed allusions to other well-known themes of the Greek lore: the Golden age, the kingdom of Cronus, the Earth-born Men (gegeneis)... My aim is to reassess the specificity of this Platonic mythopoeia setting it in the general context of ancient religion. In the first part I characterise the motif of the absence of God against the background of the phenomenological -and historical - category of the deus otiosus (with examples derived from the middle-Platonic and gnostic domains). In the second part I consider the problem of the source (or sources) of evil: apparently the corporeal principle (tò $\sigma \omega \mu \alpha \tau o \varepsilon 1 \delta \varepsilon ́$ ), more properly the state of precosmical chaos. Finally, the question is raised of Plato's dualism in this context: whether radical or mitigated.

\section{INTRODUCCIÓN}

Los estudiosos de Platón generalmente ponen de relieve que «dios» (ó $\theta \varepsilon o ́ s)$ no tiene preeminencia ontológica en el sistema de Platón'. Esta opinión se halla de hecho bien basada en la doctrina de Platón según la cual cada entidad está ontológica y axiológicamente subordinada a las Ideas, in primis la idea de Bien. Incluso en el Timeo Dios puede ser considerado como «factor of rational causation» ${ }^{2}$ que se fija en las Ideas para su actividad demiúrgica. Siendo, de este modo, indulgentes con la verdad dialéctica, no negaremos que

1 Cf. por ejemplo Mohr 1985, pp. 15-18 y A. Bortolotti, La religione nel pensiero di Platone dai primi dialoghi al Fedro, Florencia 1986, esp. pp. 15 y 234.

${ }^{2}$ Cherniss 1954 , p. 254 . Cf. Mohr 1985, pp. 22-23: «If the Demiurge bears a resemblance to any of the gods of the Western theistic traditions, it is to the god of the ontological argument, or at least of those variants of the argument, like that in Descartes's Third Meditation, which employ the principle of sufficient reason». 
el dios del Timeo y el dios del mito del Político, así como - mutatis mutandis - los dioses de las Leyes (libro diez) son personajes majestuosos cuyo papel puede parecer análogo al desempeñado por Dios (esto es, el Ser Supremo) en una religión positiva. Así que puede ser interesante para quienes se interesan por la historia de las religiones abordar un dios platónico (en este caso el dios del mito del Político) de la misma manera en que se tratarían otras realidades cultuales de la religión antigua.

\section{Dios. Su CARACTERIZACIÓN DESDE Un PUNTO DE VISTA COMPARATIVO}

Si nos preguntamos qué clase de dios es el que actúa en el escenario de este embarazoso mito, encontraríamos que es una figura de múltiples aspectos. Primero, aparece en escena con los rasgos familiares y tranquilizadores de Zeus. El dios (ó $\theta \varepsilon o ́ s)$ que «por ofrecer una prueba en favor de Atreo» es responsable «del cambio del ocaso y del orto del sol y de las estrellas» (269a 1-5) es obviamente el dios del cielo y cabeza suprema del panteón Olímpico. Según una tradición mítica atestiguada por primera vez en Eurípides ${ }^{3}$, Zeus hizo que el sol volviera atrás en su curso para demostrar la prioridad de una reclamación correcta, incluso a costa de perjudicar el orden cósmico; en otras palabras, para validar la espantosa máxima fiat iustitia, pereat mundus ${ }^{4}$. Podemos admitir que «Plato's real theological beliefs had little in common with the ancient religion» ${ }^{5}$; pero tal Zeus, tan impaciente como para interferir en los asuntos humanos y cósmicos, parece realmente simpático para el intransigente fundamentalismo ético de Platón.

Prima facie, el dios providente (ó $\theta \varepsilon o ́ s)$, que (269c 4-5) ayuda a guiar el

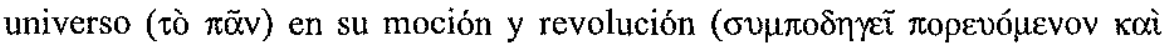
$\sigma v \gamma \kappa \cup \kappa \lambda \varepsilon \tilde{i})$, parece emparentado con el dios vigilante de la saga argiva. Si de hecho es verdad que los gérmenes del mito del Político se encuentran en una tradición presumiblemente muy antigua, Platón opera un significativo cambio de énfasis. En la época de Atreo, el cambio de dirección del sol tuvo lugar

${ }^{3}$ Cf. Adam 1891, p. 445. Hay un relato detallado de tradiciones variantes en J. G. Frazer, Apollodorus, The Library, II (Londres-Nueva York 1921), pp. 164-167, n. 1. Un comentario de Vidal-Nacquet 1975, pp. 369-70 («Platon n'avait nul besoin d'évoquer ces inquiétants "pasteurs" qu'étaient Atrée et Thyeste...») evidencia las idiosincrasias de su hermenéutica.

${ }^{4}$ Este concepto euripídeo y platónico de la Justicia de Zeus parecería característicamente no-griego si generalizáramos las conclusiones de H. Lloyd-Jones, The Justice of Zeus, Berkeley-Los Ángeles-Londres 1971, pp. 161-2: «Dike means basically the order of the universe, and in this religion the gods mantain a cosmic order. This they do by working through nature and the human mind, and not by means of extraneous interventions...»

5 Lloyd-Jones, The Justice... cit., p. 163. 
siguiendo la activa intervención del dios. En la fantasía de Platón el cambio en el movimiento del todo (que conduce a cambios del movimiento de las partes, in primis el sol y las estrellas) ocurre en el preciso momento en que el dios deja el timón, abandonando el mundo a su suerte (269 c 5). Así el dios de Platón parece mantener sus distancias con el Zeus de la religión griega estándar.

Examinemos ahora las actividades que el dios desarrolla en forma positiva (su intervención en este mundo), dejando para más adelante las actividades que desarrolla en forma negativa (su no-intervención). El dios actúa sobre el mundo en

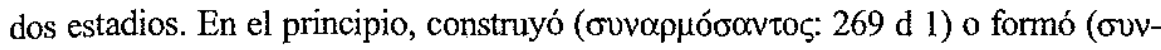

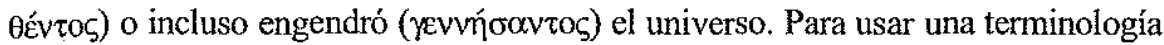

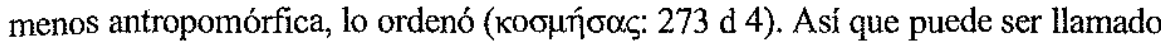
«hacedor, creador» ( $\delta \eta \mu$ tovpyós: 270 a 5) e incluso «creador y padre» (273 b 1-2). Durante el primero de los períodos alternativos del mundo, Dios acompaña al mundo en su revolución hacia adelante ( 269 c 4-5, citado supra), en otras palabras,

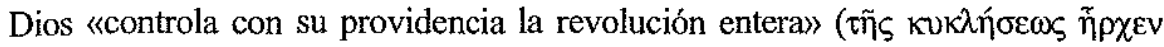

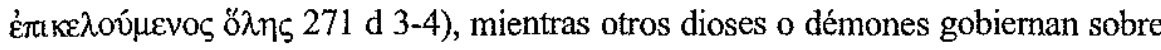
todas las partes del cosmos que se les había encargado ( $271 \mathrm{~d} 4$ - e 1). El famoso rey Crono de la leyenda era precisamente uno de los dioses acompañantes y estaba encargado de los hombres (es decir, de los varones adultos sin mujeres ni hijos) como superintendente y $\left\langle\right.$ paston ${ }^{6}$. Consecuentemente, dios es correctamente lla-

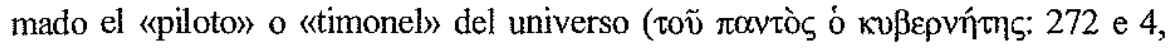
cf. 273 c 3) ${ }^{7}$.

Pasemos ahora a las actividades negativas de Dios, esto es, el momento de su no injerencia en los asuntos cósmicos. Cuando los ciclos de tiempo que le han sido señalados llegan a término, Dios abandona el universo a su suerte ( $\dot{\alpha} \vee \tilde{\eta} \kappa \varepsilon v: 269$ c 5), con el resultado de que éste comienza a girar en la dirección

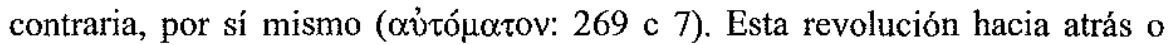

6 Para Rosen 1979, p. 74 (cf. 82) el Demiurgo y Crono son uno y el mismo igual que ocurre con Zeus: "We have three different aspects of the same deity, or in a sense, a kind of Hegelian synthesis of the two opposites, Cronos and Zeus»). Eso puede ser verdad en un nivel metafísicamente más profundo, pero va más allá de las intenciones de Platón que en este caso no parecen ser alegóricas en absoluto.

7 De acuerdo con Adam 1891, p. 446, las actividades del demiourgos y del kybernetes son en un último análisis coincidentes, esto es, «the forward revolution of the universe... means nothing more or less than the creation of the world, i.e., its ordering out of chaos». Según Mugler 1960, p. 170 (cf. pp. 173, 178 y 192) — una aproximación drásticamente reduccionista - el dios del mito del Político es un símbolo «pour l'information structurale du monde et le pouvour régulateur qu'elle exerce sur les forces diffuses de l'ananke». Para el problema del Demiurgo como un personaje real o como mero símbolo véase el destacable tratamiento de Robinson 1967, pp. 57-61, con el que coincido en lo fundamental. Para paralelos del Timeo, cf. Frutiger 1930, p. 204; Robinson 1967, pp. 58 y 61. 


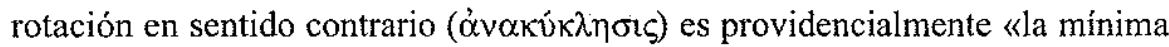
alteración posible de su propio movimiento» (269 e 2-3). Elaborando la metáfora náutica, Platón representa el comportamiento del dios como el de un timonel que se retira de la caña del timón de su barco. No hemos dicho las razones de la marcha del timonel. Dado que no está condicionado por ninguna necesidad (ananke o heimarmene) sino sólo por la naturaleza de sus modelos (las Ideas) podríamos preguntarnos si está aburrido, fatigado, inquieto o ... dormido. Puede parecer una cuestión académica, pero de hecho atañe al punto crucial, esto es, el problema de la libertad y/o responsabilidad del Dios en el gobierno de este mundo. Lo siguiente es la dramática formulación de la respuesta de Platón al misterio de la actitud de Dios vis-à-vis del mundo. Al final,

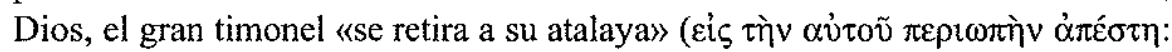
$272 \mathrm{~d} \mathrm{5})^{8}$. Esta distante actitud del Demiurgo, que tiene tan desconcertantes efectos en el macrocosmo, tiene también su impacto en el microcosmo humano. Tan pronto como el mundo es abandonado por el dios supremo, los hombres quedan privados del cuidado del demon (que presumiblemente debe identificarse con Crono) que los había tenido a su cargo y cuidado (274 b 5-6) y dejan también de ser objeto de la vigilancia a que estaban sometidos por los otros dioses inferiores (274 d 3-4).

¿Qué interpretación histórico-religiosa debe darse a este mito de una retirada alternativa ${ }^{9}$ de Dios del gobierno del mundo? ${ }^{10}$ Algunos estudiosos dudan de que esta historia pueda tomarse al nivel más serio, al menos al más alto nivel filosófico ${ }^{1 !}$. Tal escepticismo es injustificado, pues este mito (como otros mitos) no

\footnotetext{
${ }^{8}$ Compárese con la fórmula similar, pero no idéntica, del Timeo 42 e: cuando el De-

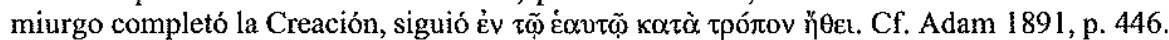

9 Según la visión cíclica del tiempo de Platón (pace Mugler 1953, pp. 93, 131; 139-143, cf. Herter 1958, pp. 328-29 y Gaiser 1963, p. 393 n. 178), al final de cada ciclo de revoluciones hacia atrás, dios «toma de nuevo su puesto en la caña del timón» (273 e 1) para restaurar las cosas que tomaron el camino de la enfermedad y la disolución durante el tiempo en que el universo estuvo abandonado a su suerte.

10 Para intentos de interpretación filosófica ver Stewart 1905, pp. 193-194; Fraccaroli 1911 , p. 84; Herter 1958, pp. 325-26; Mugler 1960, pp. 188-189; Rosen 1979, p. 80. Brisson 1992, p. 4 enfatiza que «même lorsqu'il n'intervient pas directement, le dieu reste "a la vigie"s. Pero esta reintervención en el timón del cosmos (273 e 1-4) no tiene lugar antes de que se cierre el presente período (el de Zeus). Durante este período - que es nuestro eón histórico- Dios no está al timón del universo (pace Brisson 1992,8 y passim), que se

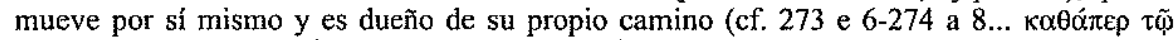

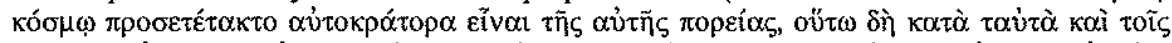

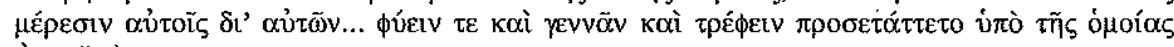
$\dot{\alpha} \gamma(0 \gamma \tilde{\eta} \varsigma$.)

II Desde Zeller, cf. especialmente Frutiger 1930, pp. 13, n. 2;14, n. 1; 52; 167-68; 219; 220 , n. $3 ; 279 ; 282$, y Taylor 1961 , pp. 208-209. Más razonable es la interpretación de Stewart 1905, pp. 193-194; Herter 1958, p. 318. Contra la «overwhelming tendency in Platonic scholarship to take the figure of the Demiurge non-literally» compartimos las
} 
contiene verdad dialéctica en sí mismo, sino conduce a la verdad en forma protréptica y analógica ${ }^{12}$. Sin embargo, relacionado con la vieja tradición como debe estarlo (cf. 269 b y 271 b), Platón produjo una tradición mítica por sí mismo (cf. $269 \mathrm{c}$ ) para sus propios fines. El impacto de Platón como creador de mitos en las posteriores doctrinas escatológicas, psicológicas, cosmológicas es bien conocido en el caso del Fedón, el Fedro, y el Timeo, pero también el mito del Político tuvo alguna reverberación sobre ciertos entornos ${ }^{13}$.

Por ejemplo, el tema del efecto «cosmético» que el dios produce a su regreso en el mundo decadente puede encontrar un eco significativo en el sueño de Virgilio del regreso de la edad de oro:

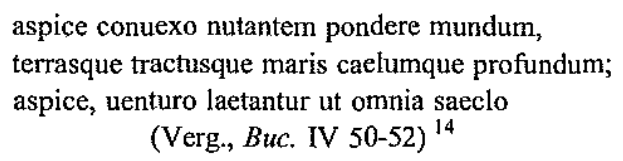

En Virgilio tenemos la misma correspondencia entre macrocosmo y microcosmo que en Platón: como resultado del cambio (inicialmente vacilante) de dirección del cosmos (mundus), tendrá lugar una restauración general de la condición del mundo (cf. v. 6 iam... redeunt Saturnia regna).

Lo que es más destacable en el mito del Político, sin embargo, es la imagen del «dios ausente», dado que en la visión platónica el período del retiro del dios es nuestro tiempo presente, en el que tiene lugar el curso de los acontecimientos históricos ${ }^{15}$. Este concepto platónico de la ausencia de Dios del gobierno del mundo es elaborado de forma original por el platónico medio Numenio (siglo Il después de Cristo). Distingue entre un Primer Dios (que

agudas notas de Mohr (1985, p. 40): «These diverse strategies should be seen... largely as (unneeded) charitable attempts to distance Plato's thought from Christian thought and more generally as attempts to reduce the number of unfashionable theological commitments in Plato's cosmology.)

12 En otras palabras «der Mythos ist der Wahrheit gegenüber ebenso vorläufig wie der Logos des Gesprächs, fuhrt aber auf andere Weise, durch abbildhaft -umfassende Wiedergabe, an die Wahrheit heran» (Gaiser 1963, p. 416, n. 259). Cf, en general Gaiser 1963, pp. 287-89: «Platon kann die althergebrachten Mythen übernehmen und seiner eigen Darstellung zugrundelegen, weil sie aus einer Zeit stammen, in der die Menschen noch die Möglichkeit der unmittelbaren Kommunikation mit dem Göttlichen hatten. Anderseits vermag er aber die Tradition von der philosophischen Prinzipienlehre aus erst grundsätzlich $\mathrm{zu}$ verifizieren») (p. 288).

13 Para la fortuna limitada del Político en los medios filosóficos véase Taylor 1961, p. 249.

14 Cf. Grilli 1983, pp. 299-300. Estoy de acuerdo con este estudioso en que esta concepción no debería confundirse con la epanodos, anakyklosis o palingenesia pitagórica (cf. Porph. V.P. 19, que debería integrarse en Dicearco fr. 33 Wehrli). Cf. también Bianchi 1988 , pp. 12-13.

15 Cf. Petrément 1947, p. 48: «Le Dieu suprême, pour Platon, est un Dieu lontain.» 


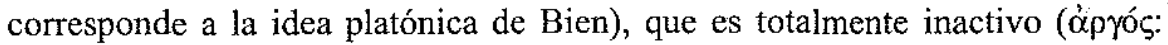

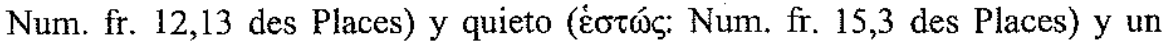
Segundo Dios (que corresponde al Demiurgo de Platón: Num. fr. 12, 2.14 des Places) que creó el mundo (Num. fr. 16,11 des Places). Pero ni siquiera el Segundo Dios, ontológicamente inferior, puede permanecer durante mucho tiempo en el nivel de las cosas creadas. Se confina a sí mismo a animar a los

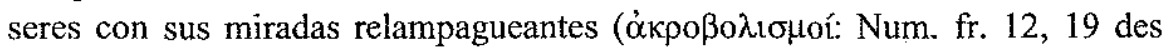
Places), pero finalmente regresa a su puesto de observación ( $\mu \varepsilon \tau \alpha \sigma \tau \rho \varepsilon ́ \phi o v \tau o \varsigma$

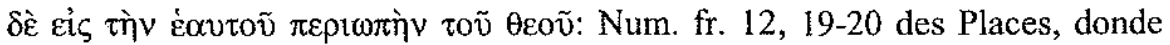
la imitación de Platón es patente, incluso en su literalidad), en orden a consa-

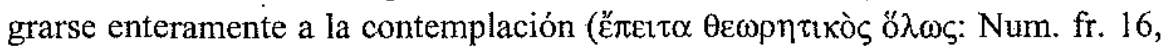
12 des Places) ${ }^{16}$.

El gnóstico Justino es un contemporáneo o epígono del platónico medio Numenio ${ }^{17}$. En el mito narrado en su Libro de Baruch el Primer Dios de Numenio (la idea de Bien) corresponde al Uno sobre todo de Justino, llamado el Bien ( $\dot{\alpha} \dot{\alpha} \gamma \alpha \theta o ́ s$ ), en un punto identificado con el Priapo cósmico; mientras que el Segundo Dios de Numenio (el Demiurgo) encuentra su pendant en el Padre de todas las cosas creadas de Justino, llamado Elohim. El Bien, aunque

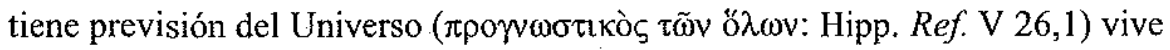
completamente aparte en un palacio de luz en el límite superior del cielo (Hipp. Ref. V 26, 15). Elohim, una vez que ha creado el mundo mediante la satisfacción mutua que sigue a su copulación con Eden (= la Tierra, la Materia o más bien el alma del mundo), desea «ascender a lo más alto de los cielos y ver si falta algo en la creación» (Hipp. Ref. V 26, 14). Por último, caído bajo el control del Bien, Elohim ya no puede o/y no quiere bajar a Eden (metáforas aparte, está decidido a dejar a mundo a su suerte: Hipp. Ref. V 26, 20).

Los dioses de Justino - el primero, ausente, el otro retirado del mundoson representantes típicos de la divinidad tal y como es concebida en una ideología gnóstica: desconocida, remota o apartada ${ }^{18}$. Más específicamente, tanto Elohim como el Demiurgo de Platón en el mito del Politico (así como, en menor grado, su contrapartida en el mito del Timeo) abandona su creación

\footnotetext{
16 Véase la bibliografía discutida por E. des Places en Numenius, Fragments, París 1973, pp. 10-14, y G. Reale, Storia della filosofia antica, IV Milán 19813, pp. 416-22.

17 Cf. S. Lilla, s. u. «Giustino gnostico» en A. Di Bernardino (ed.), Dizionario patristico e di antichità cristiane, Casale Monferrato, II, pp. 1633-34. Para un análisis más detallado y bibliografia véase J. Montserrat-Torrents, «La philosophie du Livre de Baruch de Justin», en $S P 18$, 1, ed. E. A. Livingstone, Kalamazoo (Mi) 1985 (pero 1986), pp. 253-61 y G. Casadio, «Donna e simboli femminili nella gnosi del II secolo», en U. Mattioli (ed.), La donna nel pensiero cristiano antico, Génova 1992, pp. 305-329, esp. pp. 307-308.

${ }^{18}$.Cf. Petrément 1947, pp. 47 y 160-163 «Cette étrange religion gnostique est un athéisme en ce qui conceme le monde. Le Dieu qu'on venere est un Dieu séparé du monde, et le monde se développe sans lui» (p. 163).
} 
tras haber cumplido su tarea. Así, desde un punto de vista comparativo más amplio, el dios gnóstico y el platónico pueden ser considerados ejemplos característicos de la categoría fenomenológica de deus otiosus ${ }^{19}$. El tipo del deus otiosus se atestigua de hecho en un conjunto de testimonios vasto y extendido por el mundo, desde los cultos de pueblos iletrados y (con algunas distinciones) incluso en los panteones más desarrollados de los pueblos indoeuropeos y semíticos ${ }^{20}$. La fenomenología de los llamados «dioses elevados» fue subrayada en primer lugar por el historiador y fenomenologista de la religión luterano N. Söderblom (Das Werden des Gottesglaubens, Leipzig 1919'1, 1926²), quien señaló también la relación entre la otiositas de estos creadores y la estabilidad del orden cósmico (compárese la aparente paradoja en el comportamiento del Demiurgo de Platón frente al mundo). Los Seres Supremos de hecho son personificaciones de un orden que el hombre primitivo encuentra en el mundo. Pero el Ser Supremo no necesita estar preocupado por él, ya que todo se ha organizado perfectamente $a b$ antiquo. El pasado mítico es más importante que la situación histórica presente: como un arquetipo el uno dirige y moldea la otra. Además, el Ser Supremo, creador del mundo y fundador de las leyes y los ritos, toma la actitud de deus otiosus, esto es, de un dios que, una vez que ha definido los principios fundamentales del cosmos, pierde definitivamente todo interés en los asuntos mundanos. Este ser tiene en último término sólo un papel secundario en la vida religiosa de la humanidad. Este Ser Celeste es estático como el cielo del que corrientemente toma su nombre y en el que vive. Es un deus otiosus, que aquí y ahora sólo se anima por causa de los fenómenos celestes y meteóricos: lluvia, viento, arco iris, trueno, relámpago, rayo, rocío, niebla y granizo. Pero este Ser aparentemente impasible, lánguido y perezoso es el creador y el primer regulador del cielo, la tierra, la vegetación y la humanidad: como omnividente y omnisciente (y como guardián del orden establecido por él mismo) es también el que castiga diversas transgresiones humanas. De hecho este dios es «ocioso» sólo en comparación con el activismo de las divinidades menores. Su indiferencia hacia el género humano tras la creación es relativa y no menoscaba su autoridad y primacía sobre los demás dioses. Esta otiositas aparentemente paradójica puede, pues, pertenecer a la naturaleza esencial del Ser Supremo y ser en cierto modo el complemento de su actividad creativa. Una vez hecho el mundo y establecido

19 El caso de Elohim como deus otiosus fue ya establecido por K. Kvideland, «Elohims Himmelfahrt), Temenos 1964, pp. 68-78, esp. p. 69. Los paralelos en Platón y Numenio contradicen su intento de demostrar el origen judío de este mito gnóstico.

${ }^{20}$ G. Casadio, «El and Cosmic Order: is the Ugaritic Supreme God a deus otiosus?», en Mythology and Cosmic Order (Studia Fennica 32), Helsinki 1987, pp. 45-58; Id., «A ciascuno il suo: otium e negotium del dio supremo dalla Siria alla Mesopotamia»; SMSR 58,1992 , pp. 59-79 (con extensa bibliografia). 
el cosmos, el trabajo del creador está prácticamente terminado. Cualquier intervención posterior por su parte sería no sólo superflua sino posiblemente peligrosa, dado que cualquier cambio en el cosmos podría llevarlo a caer de nuevo en el caos. La otiositas del creador es la condición más favorable y la naturalmente más adecuada para mantener el status quo (el punto de vista de Platón es marcadamente el opuesto).

Tras esta presentación sinóptica de los datos fenomenológicos que se refieren a la tipología del deus otiosus, con especial referencia a la tradición de los pueblos iletrados, estamos en condiciones de subrayar la visión del mundo tradicional o por así decirlo, el substratum sobre el que Platón construyó su representación no tradicional.

\section{BIEN Y MAL EN EL MUNDO}

En el último análisis, Platón propone el supuesto de la actuación intermitente de Dios en este mundo para brindar una causa (mitológica, por supuesto) de la existencia del mal, tanto físico como moral, en un mundo que se supone que está gobernado por un dios totalmente bueno, incapaz de sentirse agraviado y providente (cf. Tim. 29 e-30 b). En otras palabras, el mito del Politico, como el del Timeo (especialmente 28-48) y las Leyes $X$ (especialmente 899905 ) ofrece una solución al problema de la teodicea ${ }^{21}$. Platón, al dar una razón teológica de la existencia del mal a la luz de la existencia de dios, que es bueno

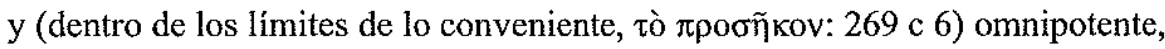
no identifica aún expresamente la causa activa del mal. A la vista de la teodicea de Platón, la causa eficiente del mal no puede ser atribuida en ningún caso ni a la acción del dios «creador y padre» ${ }^{22}$ ni a la acción contraria de una especie de dios «antagonista» ${ }^{23}$. Para Platón la verdadera fuente del mal es de hecho endógena del mundo fenoménico.

2) Cf. Stewart 1905 , pp. $193-94$ y 197-98.

22 Esta negativa, explícita en 269 e $8-9$ es implícita en el conjunto del mito al proclamar la absoluta bondad de Dios.

23 Esta posibilidad es explícitamente negada en 270 a 1-2. La negativa de la existencia de una pareja de dioses que hacen girar el mundo con propósitos conflictivos representa una negativa del dualismo Zoroástrico. Zoroastro era bien conocido por Platón (cf. Alc. I 121 1-122a; si este diálogo es de Platón: sobre la cuestión de autenticidad véase $\mathrm{A}$. E. Taylor, Plato, Londres 1926 [19607], pp. 522-23; W. C. K. Guthrie, Socrates, Cambridge 1971 , p. 150 , n. 2); y su doctrina de los principios divinos en conflicto encuentra un destacado eco en dos de los más estrechos colaboradores de Platón: Eudoxo (fr. 341 Lasserre: discusión con bibliografía en F. Lasserre ad loc, pp. 254-56) y Aristóteles (Fr. 6 Ross: cf. M. Untersteiner, en Aristotele, Della filosofia, Roma 1963, pp. 81-86; Gaiser 1963, pp. 24344 y 400 n. 209). La crítica de la doctrina zoroástrica de Ohrmuzd y Ahriman implica en cierto sentido una recepción consciente o semiconsciente de sabiduria tradicional persa. 
Revisemos ahora las fórmulas que emplea Platón en el mito del Político para identificar la fuente (o fuentes) del desorden cósmico que en último término coincide con el mal. Según Platón, el desorden se simboliza por el mo-

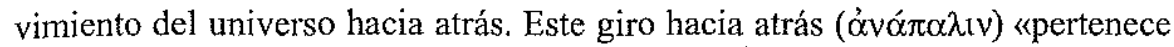

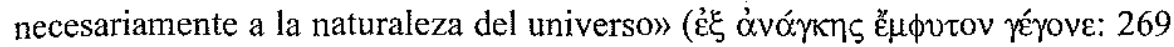

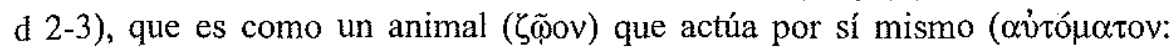
269 c 7). El universo ha sido, por supuesto, creado bueno por el Demiurgo,

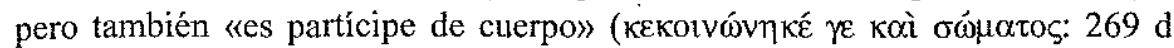
9-10). Como consecuencia de ello, el cosmos, cuando se le deja y «se mueve

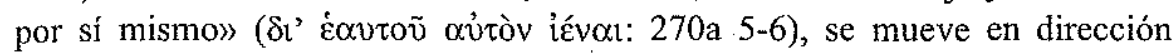
contraria (270 a 6-7). La razón por la que el cosmos comienza a moverse hacia atrás se afirma una vez más sin ambigüedades: había «un impulso (o incita-

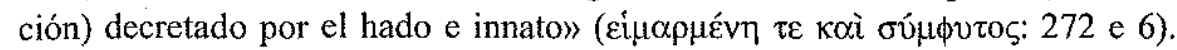
La fuente última de este impulso reside en un factor que es «algo criado con

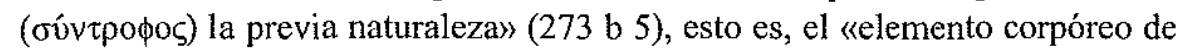

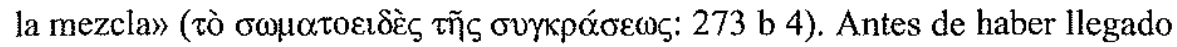
al orden presente, la naturaleza de las cosas de hecho "participaba de un

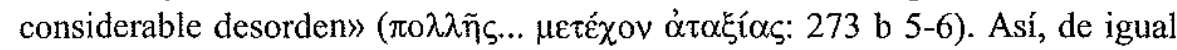
modo que todas las cosas buenas de este mundo derivan del Demiurgo, todas las crueles y perversas proceden de este previo estado de ataxia (273 b 7-c 1). Por tanto, tras la separación del universo de su creador, crece el «olvido» $(\lambda \hat{n} \theta \eta$ : 273 c 6) cada vez más en él, hasta que prevalece «el influjo de la

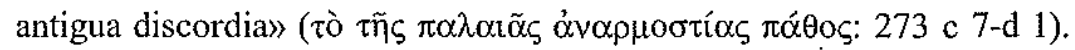

Sobre esta cuestión, largamente debatida, véase esp. Goodrich 1906 (¿recepción vía Empédocles que recibe al menos una sugerencia «from the same Persian dualism»?); Reitzenstein 1926, pp. 34-35 (Platón recibe de Irán un número de motivos, «ohne doch denschroffen Dualismus der iranischen Lehre anzunehmen»), Frutiger 1930, p. 243 y n. 4 (prudente); Schuhl 1932, pp. 57-58 («ces questions d'influence sont délicates et souvent obscures»); Bidez 1945, pp. $72-77$ y 97 («Une forme de dualisme qui ne s'est pas constituée sans une certaine notion de celui de l'Iran»); Festugière 1947, pp. $77-78$ (Platón no ignora el dualismo iranio y toma algunos rasgos de él, pero «il ne doit qu'a lui seul la maniere dont il conçoit et formule le probleme du mal, puisque cette formule, et c'est la son mérite, n'est pas de l'ordre du mythe, mais proprement et purement philosophique»); Adorno 1955, p. 137 n. 2; Mohr 1985, p. 152. Del lado de la investigación irania ver esp. J. DuchesneGuillemin, Ohrmazd et Ahriman. L'aventure dualiste dans l'antiquité, París 1953, p. 100, que destaca pertinentemente que en el mito de Platón «contrairement a la doctrine iranienne, la succession est cyclique, éternelle», e id,, Les religions de l'Orient Ancien, París 1957 (trad. ital. Catania 1960), p. 180: «Platone allude forse alla dottrina iranica, ma solamente per confutarla». Pese a su filiación platónica, Plutarco (de Is. et Osir. 45, p. 369 c; 49,

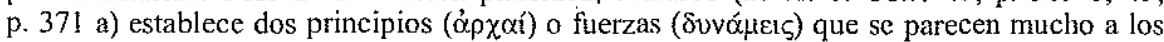
dos dioses persas antitéticos (como Plutarco admite explícitamente: de Is. et Osir. 46-47).

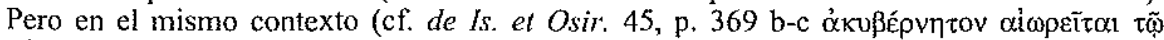

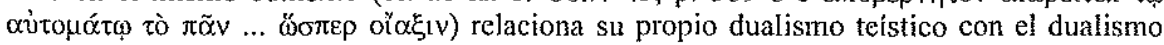
no teístico de Platón del mito del Político (cf. Hager 1962, p. 81; Bianchi 1986, p. 115). 
Así, por lo menos en el mito del Politico, la fuente última del mal se iden-

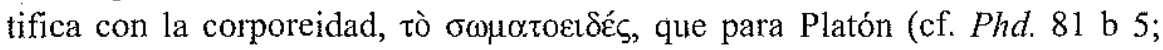
Resp. VII 532 c 7; Tim. 31 b 5) es virtualmente equivalente de la realidad fenoménica. Pero ni lo corpóreo es tan coincidente con el mal, ni el mal se origina de lo corpóreo in se ${ }^{24}$. El mal deriva más bien de un factor que es originariamente inherente a la realidad precósmica - la ataxia ${ }^{25}$ o anarmostia ${ }^{26}$ inherente al caos original ${ }^{27}$.

${ }^{24}$ Cf. Robinson 1967 , p. 83 «It can mean simply that in any complex involving body or the bodily, results tend to be less perfect than in a complex which does noty.

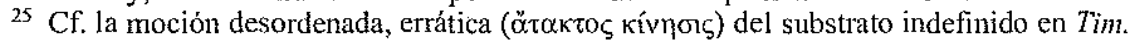
30 a y del universo guiado (pero es una hipótesis que implica una reductio ad absurdum, cf. Festugière 1947, p. 73 y Taylor 1961, p. 210) por un alma mala en Leg. X 897 d 1 y 898 b $5-8$.

26 «Discordancia» o «disonancia», que es lo contrario de $\dot{\alpha} p \mu o v i ́ \alpha\left(=\dot{\alpha} \rho \varepsilon \mathfrak{r}^{\prime}\right)$ y virtualmente coincidente con el mal ( $\alpha \alpha \kappa i \alpha)$ : Phaed. 93 e 6 (un parecer pitagórico, por supuesto).

${ }^{27}$ Cf. Fraccaroli 1911 , p. 83, Reinhardt 1927, p. 272, Frutiger 1930, p. 189 («le mouvement inverse ou spontané est, avec toutes ses conséquences facheuses, l'heritage du désordre originel, de l'état antérieur a l'activité du démiurgen), Diès 1935, pp. XXXXXXII, Bidez 1945, n. 15, Pétrement 1947, pp. 45-47 («le mouvement, cet ordre naturel, qui n'est aux yeux de Platon qu'un désordre, paraît bien être pour lui la cause du mal»), Festugière 1947, pp. $71-72$ y 74 (la fuente del mal es la snature corporelle avant que le monde n'ait été organisé en Cosmos»), Cherniss 1954, pp. 255, n. 21 y 259, n. 44 (véase la afirmación más general, p. 257: «Positive evil in the world, both absolute and relative, is produced by the misguided motion of evil souls» y cf. la bibliografia sobre el terna más controvertido de la(s) fuente(s) del mal, p. 253, n. 1), Adorno 1955, pp. 140-141, Herter 1958, pp. 319-23 («so darf man schliessen, dass die Weltseele von dem Drange ihres Körpers zur Umkehr irgendwie infiziert ist»: p. 32.2), Mugler 1960, pp. 167, 169, 174 («le sens rétrograde est produit par les mouvements non contrôlés de la matière, par l'automaton du flux materiel qui déborde ses digues»), 175, 177, 180 (fuerza de necesidad = fuerza de

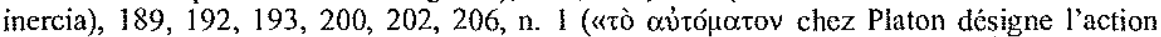
des forces non canalisées de la nécessité»), Taylor 1961, pp. 209-210 (el Político, como el Fedón «suggests what became the tenet of popular Platonism in later antiquity, that "body" or "matter" is the source of evil». Pero esto contradice la enseñanza de Leyes $896 \mathrm{~d}$ sobre almas como fuentes últimas de todo movimiento, cf. Cherniss supra), Hager 1962, p. 74 (el mito del Político ofrece «die deutlichste Belegstelle fur die Materie als Prinzip der

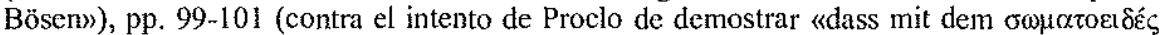

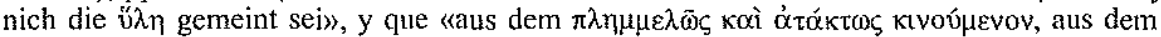

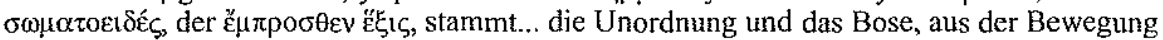
der Materie, nich aus der Materie selbst», sostiene enérgicamente que «die Spaltung, die

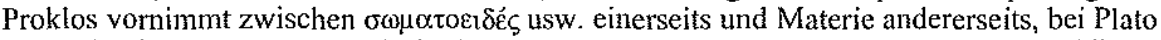
selbst durch nichts gerechtfertig ist»), y cf. pp. 73-75 para un status quaestionis con bibliografia sobre el problema de la fuente del mal segín Platón, Robinson 1967, pp. 58 y 62-64 ( «the "bodily" which accounts for the evil in the world ... has some affinities with the Necessity of the Timaeus», y en este caso «the evidence of the text seems to point to Plato's discomfort about the whole matter», 63), Mohr 1985, pp. 141-157 («the corporeal on its own without psychical influences moves chaotically, is the efficient cause of the retrograde cycle of the universe, and is a positive source of disorder and evil» [p. 156], mientras «the World-soul itself, seems not primarily to be viewed as an efficient causa either of motion as such or of motion as regular or erratic» [p. 157]; ver p. 142 para una discusión de la bibliografia sobre las causas del «disorderly motion» en el Politico), Brisson 1992, p. 4 
Si ahora suscitamos la pregunta de si «era Platón dualista en el mito del Politico», contestaríamos sin duda afirmativamente. En este mito Platón postula de una forma inequívocamente dualista dos principios opuestos que subyacen como la raíz de todos los fenómenos del universo. Estos principios eran evidentemente el Demiurgo, de una parte, y la condición caótica precósmica del universo, de otra. Ambos tienen reflejos en el nivel ético -el Bien y el Mal, respectivamente- Pero al mismo tiempo son principios ontológicos y causas primarias o secundarias de todos los fenómenos que existen en el universo y determinan su naturaleza ${ }^{28}$. Podemos admitir incluso que el principio divino tiene el status ontológico más alto y así esto parece ser un dualismo relativo o mitigado en el que un principio es subordinado al otro. En este sistema, sin embargo, el Demiurgo (el ordinator activo) y el Caos (el ordinandum pasivo) «are and remain opposed to one another and cannot be derived from or reduced to each other» ${ }^{29}$. Los dos principios parecen ser también coeternos, en el sentido de que ambos existen y actúan desde el principio. Si insistimos, como hace U. Bianchi ${ }^{30}$ en la coeternidad de los principios, pasan-

(ule renversement de la révolution cosmique s'explique par le mouvement de l'âme du monde qui se trouve, elle, sous l'influence du corps»).

${ }^{28}$ Era también la opinión de Aristóteles (Metaph. I 6, 988 a 7-17), que señala en el sistema de Platón dos principios ( $\sigma \tau \iota\llcorner\chi \varepsilon i \alpha)$ que representan el Bien y el Mal. Cf. la aguda crítica de Hager 1962, pp. 75-76: «Das materiell körperliche kann nach unserer Platointerpretation niemals dem ordnenden Geistigen als gleichberechtige Wesenheit und gleich mächtiges Aufbauprinzip dem des Alls zur Seite treten... Genau so wenig wie das Materialprinzip den Geistprinzip gleichberechtigt gegenübertritt (und dies allein wäre wahrer Dualismus) genau so wenig tritt nach Plato auch das Böse dem Guten gleichberechtigt gegenüber». Para otras referencias al dualismo de Platón visto por Aristóteles véase Gaiser 1963, pp. 243 y 400, n. 209. Entre los modernos abogados del dualismo en Platón con referencia al Político, cf. Pétrement 1947, p. 118: «Ainsi le corps, ou plutôt le principe corporel, sans être le mal, est le principe du mal», Festugière 1947, p. 68: «Le dualisme platonicien est l'opposition d'un principe du bien et de la matière», y esta «dualité du bien et du mal se retrouve dans le mythe fameux du Politique» (p. 71), Gaiser 1963, pp. 212, 243-44, 284 («der zeitlose Gegensatz der Grundprinzipien, Peras und Apeiron»), 391, n. 173 («die Spannung zwischen Nus und Epithymia in der Seele ergibt sich aus der Mittelstellung zwischen Idee und korperlicher Erscheinung, Einheit und unbestimmter Vielheit»), Bianchi 1986, pp. 115-16 y n. 19, definiendo el dualismo platónico como «dialettico e, misuratamente, procosmico»: Id. 1992, pp. 51-52: según Platón, el mal tiene un carácter ontológico en el contexto de un sistema dualista, «essendo espresso come un prestablito defectus di valore a carico dei livelli inferiori dell'universo", y pese al hecho de que no descubrimos la existencia udi una sostanza attiva o maligna, una sostanza a tutto tondo, una sostanza per cosi dire "in convesso").

${ }_{29}$ P. F. M. Fontaine, The Light and the Dark. A Cultural History of Dualism, III, Amsterdam 1988, p. 180 (para un tratamiento interesante -aunque en algunos casos superficial - del tema general del dualismo de Platón véanse pp. 119-132).

${ }^{30}$ S. u. «Dualism», en M. Eliade (ed.), The Encyclopedia of Religion, Nueva York-Londres 1987 , IV, pp. 506-512, 507-508. 
do por alto el hecho de que no son iguales, podemos aun así considerar que el dualismo de Platón es radical, pese a que podría producirse entre muchos eminentes platonistas el rechazo de tal aserto.

Hay otro aspecto del mito del Político que es de mayor relevancia para la historia de las religiones. Debe reconocerse en su relación con la historia de la humanidad, con las ambigüedades intrínsecas a su curso (la idea de progreso y/o regreso que se reflejan en las leyendas de los gegeneis, la Edad de Oro y el reino de Crono). Pero todo este intrigante asunto ${ }^{3 !}$ no puede ser debatido ahora, dada la perspectiva más estricta de este artículo.

\section{Giovanni CASADio}

\section{BIBLIOGRAFÍA}

Adam, J. (1891): «The Myth in Plato's Politicus», CR, pp. 445-46.

Adomo, F. (1966): «Dialettica e politica in Platone: saggio sul Politico e sulle Leggi», $A A T C$, pp. 97-200.

Bianchi, U. (1986): «Plutarco e il dualismo», en F. E. Brenk- I. Gallo (edd:), Miscellanea plutarchea. Atti del I convegno di studi su Plutarco (Roma 23 nov. 1985), Ferrara, pp. 111-120.

- (1988): «ll 'primo' e l' 'altrove'. Variazioni sul tema del rapporto tra dei e uomini nella religione greca antica», Kernos 1, pp. 9-17.

- (1992): «Per la storia del dualismo in Grecia», en Id., La soteriologia del cristianesimo. Ricerche storico-comparative, Roma, pp. 39-52.

Bidez, J. (1946): Eos ou Platon et l'Orient, Bruselas.

Brisson, L. (1992): «Intérpretation du mythe du Politique» (Preliminary Paper presented at the Third Symposium Platonicum), Bristol, pp.1 -13.

Chemiss, H. (1954): «The Sources of Evil according to Plato», PAPhS, pp. 23-30= Id. Selected Papers, Leiden 1977, pp. 253-60.

Diès, A. (1935): «Notice», en Platon, Le Politique, París, pp. VII-LXV.

Festugière, A.-J. (1947): «Platon et l'Orient», $R P h=$ Id., Etudes de philosophie ancienne, París 1971, pp. 39-79.

Fraccaroli, G. (1911): «Introduzione», en Platone, II Sofista e l'Uomo politico, Turín, pp. 73-85 (cap. IV).

Frutiger, P. (1939): Les mythes de Platon, París.

Gaiser, K. (1963): Platons Ungeschriebene Lehre, Stuttgart 1968 .

Goodrich, W. J. (1906): «Plato, Politicus 269e-270a.- An Allusion to Zoroastrianism?», CR, pp. 208-209.

31 Véanse las contribuciones de Vidal-Naquet 1975 y Tulli 1980. Ambas -por una razón o por otra - son poco satisfactorias desde un punto de vista histórico-religioso. 
Grilli, A. (1983): «Pitagoreismo e non nella IV egloga», en Atti del convegno virgiliano di Brindisi nel bimilleanario della morte. Brindisi 15-18 ott. 1981, Perusa, pp. 285-302.

Hager, F. P. (1962): «Die Materie und das Böse im antiken Platonismus», MH, pp. 73-103.

Herter, H. (1958): «Gott und die Welt bei Platon. Eine Studie zum Mythos des Politikos», BJ, pp. 106-117 = Id., Kleine Schriften, Munich 1975, pp. 316-329.

Mohr, R. D. (1985): The Platonic Cosmology, Leiden.

Mugler, Ch. (1953); Deux themes de la cosmologie grecque: Devenir cyclique et pluralité des mondes, París.

- (1960): La physique de Platon, París.

Pétrement, S. (1947): Le dualisme chez Platon, les gnostiques et les manichéens, Paris (reimp. Brionne 1982).

Reinhardt, K. (1927): Platons Mythen, Bonn = Id., Vermächtnis der Antike, Gotinga 1960 [19662], pp. 219-95.

Reitzenstein, R. (1926): "Vom Damdad-Nask zu Plato», en Id. y H. H. Schaeder, Studien zum antiken Synkretismus aus Iran und Griechenland, Leipzig-Berlín, pp. 3-37.

Robinson, T. M. (1967): «Demiurge and World Soul in Plato's Politicus», AJPh, pp. 57-66.

Rosen, S. (1979): «Plato's Myth of the Reversed Cosmos», RMeta, pp. 59-85.

Schuhl, P.-M. (1932): «Sur le mythe du Politique», RMM, pp. 47-58.

Stewart, J. A. (1905): The Myths of Plato, Londres, Fontwell (Sussex) 19602.

Taylor, A. E. (1961): «Introduction», en Plato, The Sophist and the Statesman, Londres, pp. 189-252.

Tulli, M. (1990): «Età di Crono e ricerca sulla natura nel Politico di Platone», SCO, pp. $97-115$.

Vidal-Naquet, P. (1975): «Le mythe platonicien du Politique, les ambigüités de l'âge de l'or et de l'histoire», en J. Kristeva (ed.), Langue, discours, société. Pour Émile Benveniste, París, pp. 374-91 = Id., Le chasseur noir, París 1981, pp. 361-80. 